\title{
The neurogenic effects of rosmarinic acid in a mouse model of type 2 diabetes mellitus
}

\author{
Mahnoor Ali' ${ }^{1}$, Saadia Zahid ${ }^{10}$ * \\ ${ }^{1}$ Neurobiology Research Laboratory, Department of Healthcare Biotechnology, Atta-ur-Rahman, \\ School of Applied Biosciences, National University of Sciences and Technology, Islamabad, Pakistan
}

\begin{abstract}
There is emerging evidence for a dysregulation of insulin signaling in the brains of patients with Alzheimer's disease (AD) with overlapping molecular features to Type 2 Diabetes Mellitus (T2DM). In addition, T2DM is a known risk factor of AD. The goal of this study was to investigate the neurogenic and neuroprotective potential of rosmarinic acid (RA) in a streptozotocin (STZ)induced combined with high fat diet (HFD) mouse model of diabetes. Animals were divided into four experimental groups (control, diabetic, diabetic + RA, RA only). Behavioral analysis was performed to assess spatial learning and anxiety levels of animals, whereas quantitative real time PCR was carried out to assess the gene expression levels of neuronal markers of neurogenesis (Ki67, DCX and NeuN). A significant decrease in memory and spatial learning was observed in the diabetic mice, which was substantially improved by RA treatment. RA also increased the gene expression of NeuN, DCX and Ki67, which were dysregulated in the diabetic model. This study proposes RA as a potential therapeutic agent to mitigate neuronal dysfunction associated with T2DM by promoting adult hippocampal neurogenesis.
\end{abstract}

Keywords: Diabetes Mellitus. Neurodegeneration. Neurogenesis. Rosmarinic acid. Streptozotocin.

\section{INTRODUCTION}

Diabetes mellitus (DM) is a multifactorial, metabolic disorder, characterized by extreme levels of glucose in the body, a condition referred to as hyperglycemia (Alberti, Zimmet, 1998). Type 2 diabetes mellitus (T2DM) afflicts 422 million people globally and is expected to become the seventh leading cause of mortality by 2030. Hyperglycemia is known to cause hypertension, cardiovascular problems and renal disease, as well as to being toxic to brain cells (Rossetti, 1995). Hyperglycemia is a consequence of insulin resistance, which generally occurs before the manifestation of T2DM. Referred to as prediabetes,

\footnotetext{
*Correspondence: S. Zahid. Neurobiology Research Laboratory. Department of Healthcare Biotechnology. Atta-ur-Rahman School of Applied Biosciences (ASAB). National University of Sciences and Technology (NUST), Islamabad, Pakistan. Phone: +92-51-90856134. Email: saadiazahid@hotmail.com/saadia.zahid@asab.nust.edu.pk
}

this state progresses to impair the normal secretion of insulin which leads to a hyperglycemic condition and ultimately the development of chronic T2DM (DeFronzo, 2004).

There is mounting evidence showing that T2DM is a risk factor for developing Alzheimer's disease (AD), which has been referred as Type 3 diabetes (Ahmed, Mahmood, Zahid, 2015; Vagelatos, Eslick, 2003). While in T2DM most of the insulin resistance occurs in peripheral regions, in $\mathrm{AD}$ the insulin resistance is limited to the brain (Moroz et al., 2008). In addition, both T2DM and AD share $\boldsymbol{\beta}$-amyloid toxicity in the brain associated with cognitive deficits (Arrieta-Cruz, Gutiérrez-Juárez, 2016). Although several potential explanations for this overlap have been proposed, such as hyperglycemia, insulin resistance and hyperinsulinemia (Sridhar, Lakshmi, Nagamani, 2015), the common underlying mechanism(s) to the disruption of the pathway(s) is/are still yet unknown. 
Rosmarinic Acid (RA) is an active constituent of Rosmarinus offi inalis L. that possesses anti-oxidative, anti-inflammatory and neuroprotective properties (Petersen, Simmonds, 2003; Khamse et al., 2015; Hamaguchi et al., 2009).). RA minimizes and improves the learning and cognitive deficits in a mouse model of ischemia (Fonteles, 2015). It is not known whether these potential therapeutic properties may also assist in mitigating the cognitive impairment that occurs with T2DM. The goal of this study was to investigate the potential neurogenic and neuroprotective effect(s) of RA on adult hippocampal neurogenesis and cognition in a mouse model of T2DM.

\section{MATERIAL AND METHODS}

\section{Material}

\section{Reagents and Chemicals}

Streptozotocin (STZ) was bought from Calbiochem, Merck (Catalogue\# 572201). Trizol was obtained from Invitrogen (Thermo Scientific, USA). The Reverse transcriptase (RT), Taq polymerase and deoxy-nucleotide triphosphate (dNTPs) were obtained from Fermentas (Thermo Scientific, USA) while the Sybr Green HOT FIREPol ${ }^{\circledR}$ EvaGreen $^{\circledR}$ qPCR Mix Plus was acquired from Solis Biodyne (Estonia). The rest of the chemicals and reagents used in this study were purchased from Sigma-Aldrich (USA).

\section{Ethics Statement}

The experiments were carried out in accordance with the regulations of the Institute of Laboratory Animal Research, Division on Earth and Life Sciences, National Institute of Health, USA (Guide for the Care and Use of Laboratory Animals: Eighth Edition, 2011). Ethical approval was also granted by the Internal Review Board (IRB) of ASAB, NUST.

\section{Animals}

Male Balb/c mice (4 weeks old) were chosen for this study and obtained from the Laboratory Animal House of Atta-ur-Rahman School of Applied Biosciences (ASAB), National University of Sciences and Technology (NUST). The animals (total $=24$ ) were housed in standard cages and maintained under standard conditions of living at constant temperature of $25 \pm 2{ }^{\circ} \mathrm{C}$ with a 12 hours light/dark cycle.

\section{Induction of Type 2 Diabetes in mice and Rosmarinic Acid Treatment}

The mouse model for T2DM was developed as previously reported (Noor, Zahid, 2017; Li et al., 2012). Briefly, shortly after weaning, the animals were allocated to different dietary regimens. To induce T2DM, animals were fed with high fat diet (HFD) (basic mice feed $59 \%$, sugar $20 \%$, animal fat $18 \%$ and egg yolk $3 \%$ ) and administered with STZ $(100 \mathrm{mg} / \mathrm{kg}$, dissolved in $0.1 \mathrm{M}$ citrate buffer) intraperitoneally at $6^{\text {th }}$ and $9^{\text {th }}$ week of age. Control mice were fed with standard chow (crude protein $30 \%$, crude fat $9 \%$, crude fiber $4 \%$ and moisture $10.4 \%$ ) and injected with citrate buffer as placebo. The FBG levels were measured in two occasions; after the first injection of STZ and then 8 days after the administration of the second dose, using the On-Call ${ }^{\circledR}$ EZ II Blood Glucose Monitoring Device by ACON International USA. Animals with FBG levels $>7.8 \mathrm{mmol} / \mathrm{L}$ were considered to have developed diabetes (Dong et al., 2013; Ito, 2008) and used for further analysis.

RA $(4 \mathrm{mg} / \mathrm{kg}$, dissolved in $0.9 \%$ saline, (Ito, et al., 2008)) was administered intraperitoneally to half of the control mice and half of the T2DM mice $(n=6$, per group), while the remaining mice were given saline only. Treatment with RA spanned a period of 7 days and upon completion, the fasting blood glucose (FBG) levels of all animals were recorded. The detailed experimental protocol is illustrated in Figure 1.

\section{Novel Object Recognition Test}

The novel object recognition test (NOR) was carried out with minor modifications in the procedure described by (Leger et al., 2013). Briefly, an open field apparatus $(70 \mathrm{~cm} \times 90 \mathrm{~cm}$ x $60 \mathrm{~cm}$ ) was used to conduct the test. Each mouse was allowed to familiarize with the apparatus in a 5 min habituation phase, and subsequently subjected to the session I, in which two identically similar objects were introduced into the box, placed equidistant from one another, and the mice were allowed to explore the objects. A 20 min interval followed before the start of session II. In this session, two dissimilar objects were introduced in the arena and mice were allowed to explore. Both sessions were video recorded and analyzed for the novel object preference, expressed as a ratio of the 


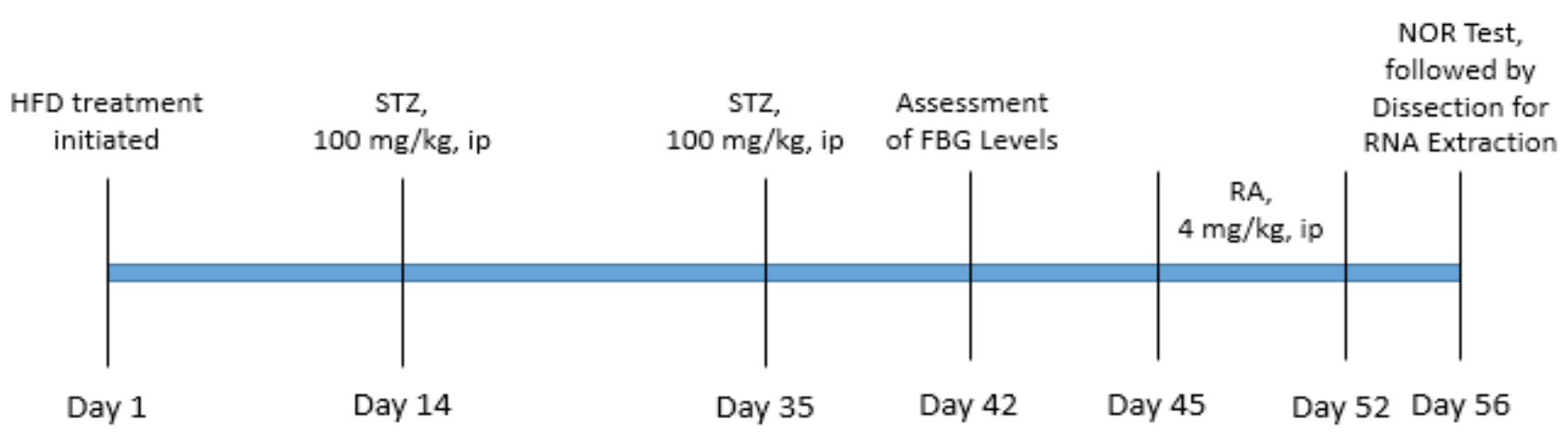

FIGURE 1 - Study Design: Timeline depicting the 56-day period for the induction of diabetes, RA treatment, memory assessment with the NOR test and animal sacrifice for mRNA expression analysis in the brain.

time spent exploring the novel object (TB x 100) / (TA $+\mathrm{TB}$ ), where TA and TB are the time spent exploring the familiar object and the novel object, respectively. Exploration of objects was considered when deliberate contact occurred or the nose of the animal was pointing toward the object at a distance $\leq 1 \mathrm{~cm}$.

\section{Gene Expression Studies}

All the animals were sacrificed a day after the completion of behavioral testing. Animals were anesthetized and euthanized by cervical dislocation. Cortex and hippocampus were immediately removed, snap frozen in liquid nitrogen and stored at $-80{ }^{\circ} \mathrm{C}$ until further use.
RNA was extracted using Tri-reagent according to a standard protocol. The extracted RNA was quantified using Nanodrop 2000 (Thermoscientific, USA) and an equal amount of RNA $(2 \mu \mathrm{g})$ was transcribed into cDNA. The quantitative real time PCR was carried out as described by Noor et al., 2017. Briefly, $4 \mu \mathrm{L}$ of HOT FIREPol ${ }^{\circledR}$ EvaGreen ${ }^{\circledR} \mathrm{qPCR}$ Mix Plus, $1 \mu \mathrm{L}$ of forward and reverse primers and $1 \mu \mathrm{L}$ of cDNA template was mied and a reaction mixture was prepared. ABI Prism 7300 sequence detection system (Applied Biosystems, 7300) was used to quantify the expression of DCX, NeuN and Ki67 using specific forward and reverse primers (Table 1). The quality of PCR product was assessed through dissociation curves and $2 \%$ agarose gel electrophoresis. All the values were normalized to $\boldsymbol{\beta}$-actin and analyzed relatively using the $2^{-\Delta \Delta C \mathrm{~T}}$ method (Livak, Schmittgen, 2001).

TABLE I - Forward and reverse primer sequences used for the expression analysis of $\beta$-Actin, NeuN, Ki67 and DCX

Gene $\quad$ Primer Sequences 5' -3 , Length of the PCR Product (bp)

GCCTTCCTTCTTGGGTATGG

CAGCTCAGTAACAGTCCGC 
TABLE I - Forward and reverse primer sequences used for the expression analysis of $\beta$-Actin, NeuN, Ki67 and DCX

\begin{tabular}{lcc}
\hline Gene & Primer Sequences 5 $\mathbf{-}-\mathbf{3}$, & Length of the PCR Product (bp) \\
\hline Ki67 & CTGCCTGCGAAGAGAGCATC & 81 \\
\hline DCX & AGCTCCACTTCGCCTTTTGG & 182 \\
\hline
\end{tabular}

\section{Statistical Analysis}

The results were expressed as mean \pm standard error of the mean (S.E.M.). Data were analyzed via one-way analysis of variance (ANOVA) followed by Bonferroni multiple comparison post hoc test for determining between-group differences. $p$ value $<0.05$ was considered significant. GraphPad Prism 5 software was used to generate the graphical representation of data.

\section{RESULTS AND DISCUSSION}

The current study examined the effects of RA on cognitive dysfunction and adult hippocampal neurogenesis in a T2DM mouse model using behavioral testing and gene expression analyses.

\section{Rosmarinic Acid Decreases Hyperglycemia in a T2DM Mouse Model}

The protocol was optimized for the generation of diabetic mice. The group treated with HFD and streptozotocin injections showed hyperglycemia (Figure 2a). The FBG levels were higher than the cut off value of $7.8 \mathrm{mmol} / \mathrm{L}$. Interestingly, the increased levels were reduced after treatment with RA (Figure 2). The HFD assisted in significantly increasing the weight of the animals whereas the small doses of STZ worked to destroy the pancreatic $\beta$ cells to produce insulin deficiency, in accordance with other studies (Zhang et al., 2008; Srinivasan et al., 2005). The classical symptoms of T2DM and the development of the hyperglycemic condition were confirmed by assessing the FBG levels in animals. The average FBG level of the control mice was $6.4 \mathrm{mmol} / \mathrm{L}$, and those of the diabetic mice were $13.6 \mathrm{mmol} / \mathrm{L}(>7.8 \mathrm{mmol} / \mathrm{L})$. This confirmed the development of T2DM in mice, similar to what had been reported in previous studies (Noor, Zahid, 2017; Dong et al.,2013). The decrease in FBG levels in the diabetic group upon RA treatment indicates a potential anti-diabetic effect (Figure 2b).

\section{Hyperglycemia Induces Cognitive Deficits}

The NOR test was used to assess cognitive decline in response to hyperglycemia induced in the T2DM mouse model. The novel object preference was calculated for each group. Animals with a higher value of preference demonstrate intact memory and recognition while those with lower preference show an inability to distinguish the familiar object with the novel one. Due to impaired memory, the diabetic mice were unable to distinguish the familiar and novel object apart, exhibiting a significantly impaired memory ( $p<$ 0.05 ) in contrast with the other groups, and therefore had no obvious preference for either of the objects (Figure 3).

Previous work suggested learning deficits in diabetic mice as well as inability to react normally to object novelty (Carvalho et al., 2013). The assessment of learning and cognitive capabilities in diabetes through NOR indicated a significant decline in the memory and learning with T2DM. T2DM animals exhibited inability to differentiate between the familiar and novel object and presented diminished reference memory. These findings 
corroborate the knowledge that cognitive deficits are a complication in T2DM. It was shown previously in a A $325-35$ mouse model that RA significantly increased the time spent with the novel object in both control and
A $325-35$ treated mice (Alkam et al., 2007). Our results also demonstrate that RA is involved in enhancing cognitive performance and preventing memory deficits that arise with T2DM.

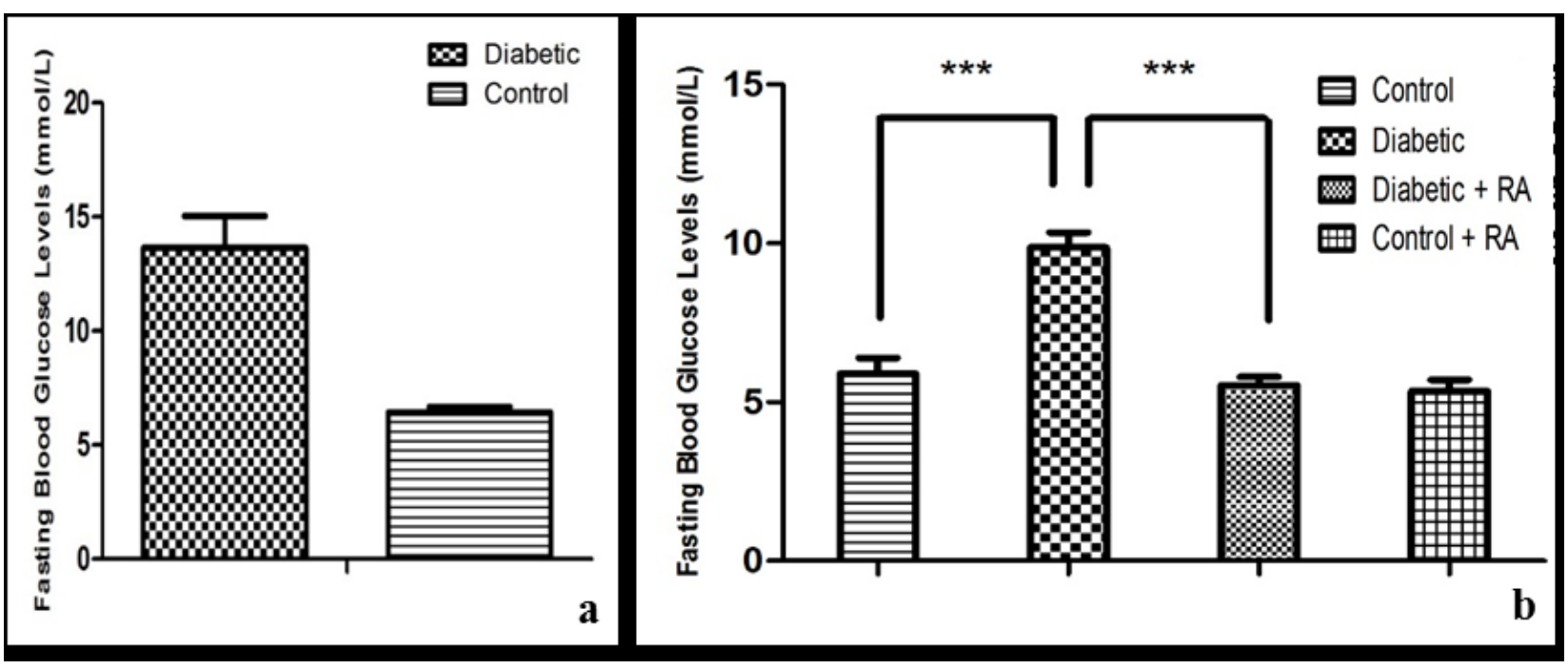

FIGURE 2 - Average Fasting Blood Glucose (FBG) Levels (mmol/L): (a) Average FBG (mmol/L) of control and diabetic groups which were kept on HFD from 4 to 9 weeks of age and injected with STZ (100 mg/kg ip) at 6 and 9 weeks of age. (b) Average FBG $(\mathrm{mmol} / \mathrm{L})$ after rosmarinic acid treatment of experimental animal groups (diabetic, control, diabetic + RA and RA only) after 7 days treatment ( $4 \mathrm{mg} / \mathrm{kg} \mathrm{RA}$ ip).

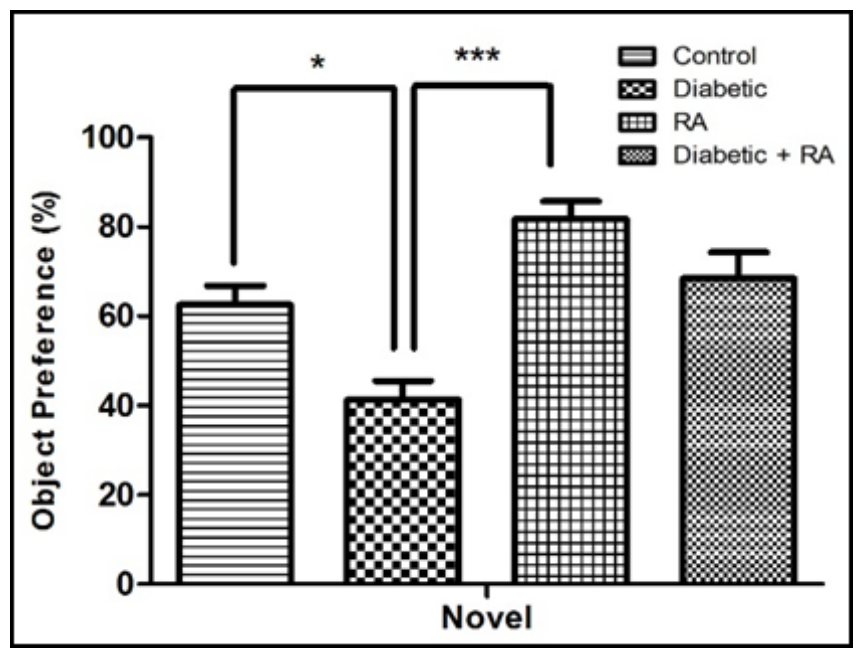

FIGURE 3 - Effects of Hyperglycemia on Memory and Cognition Using Novel Object Recognition (NOR) Test: The graph depicts the percent time spent exploring the novel object in NOR test. In comparison with the control, the diabetic groups showed a significant decline in novel object recognition which indicates compromised memory in the latter. Data was analyzed using one-way ANOVA, followed by multiple comparison Bonferroni test. The data is expressed as mean $\pm \mathrm{SEM}, * p<0.05$, $* * * p<0.0005$. 


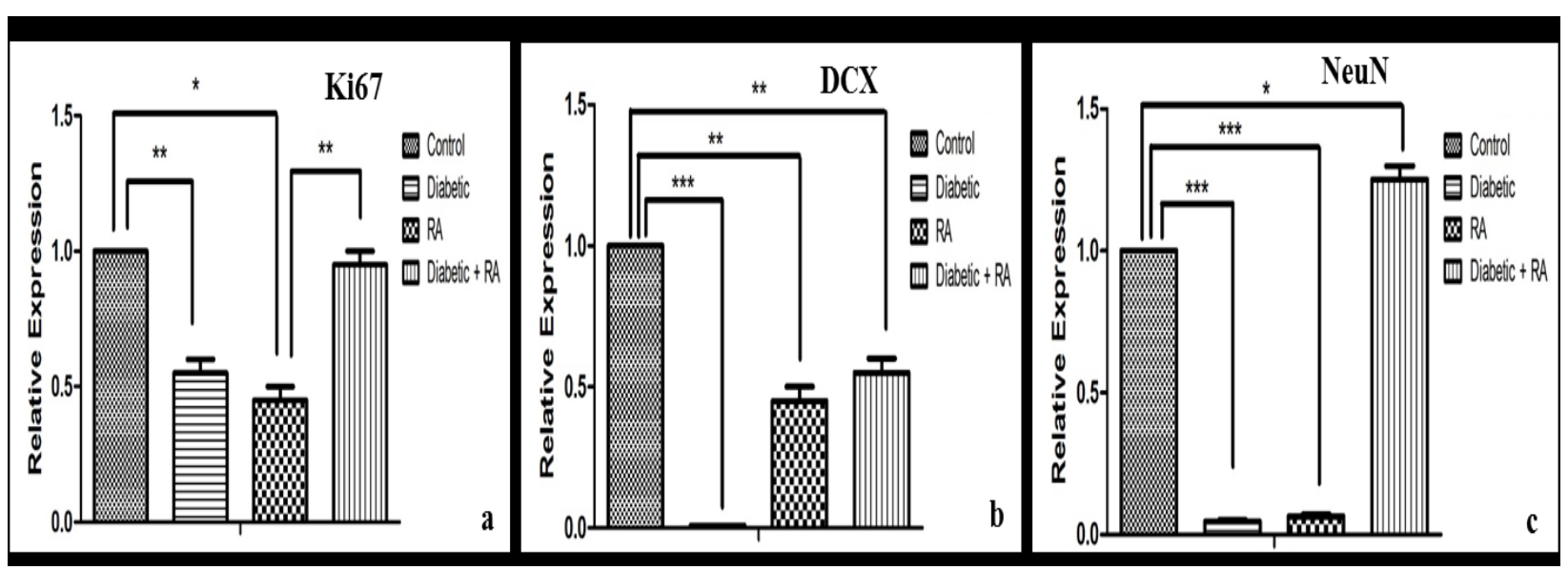

FIGURE 4 - Histogram Representing the Relative Expression of Ki67, DCX and NeuN in Control and Experimental Groups: Decline in the expression of (a) Ki67, (b) DCX and (c) NeuN in the diabetic mice shows an impaired neuronal proliferation capability. A substantial increase after the RA treatment shows its promising influence on neuronal proliferation. The data was analyzed using one-way ANOVA and is shown as mean $\pm \mathrm{SEM}, * p<0.05 * *, p<0.005$ and ${ }^{* * *} p<0.0005$
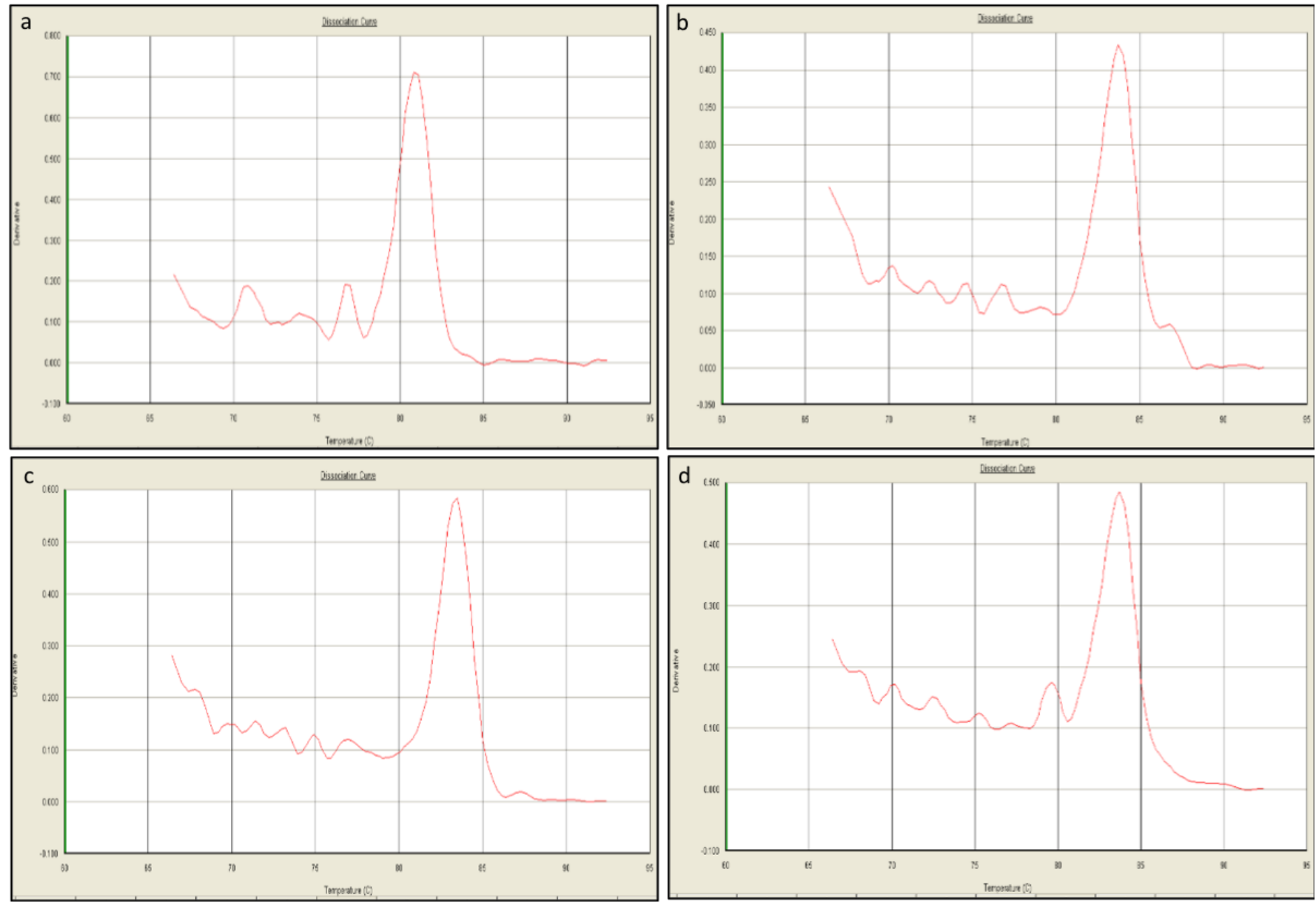

FIGURE 5 - Dissociation curves of qPCR products. The quality of PCR products a: $\beta$-Actin, b: KI67, c: DCX and d: NeuN was assessed through dissociation curves and $2 \%$ agarose gel electrophoresis. All the values were normalized for $\beta$-actin and analyzed relatively using the $2^{-\Delta \Delta C T}$ method. 


\section{Neuronal Marker Expression Analysis}

A significant decrease in the expression of Ki67, a marker of proliferation, was observed in the diabetic group as compared to the control $(p<0.005)$, indicating reduced neuronal proliferation in the animals in response to the T2DM condition. However, after the RA treatment there was a substantial increase in the expression of $\mathrm{Ki} 67$ in the diabetic group, indicating that RA may have an influence in neuronal proliferation under certain circumstances. DCX, which codes for the neuronal migration protein doublecortin, is known to be expressed by immature neurons and is thereby considered a marker of neurogenesis. A significant decline in DCX gene expression was observed in the diabetic mice in comparison with control $(p<0.0005)$, suggesting damage to neurons as a consequence of T2DM. A similar reduction in DCX gene expression was also observed in the RA treated mice $(p<0.005)$, and expression of NeuN was also significantly decreased by RA in comparison with control ( $p<0.0001)$. However, RA prevented the decrease in expression of both DCX and NeuN in the T2DM group ( $p<0.05$ ) (Figure $4-5$ ).

RA has been documented to mitigate neuronal damage caused by T2DM (Tavafi et al., 2011; Sotnikova et al., 2013) and is particularly effective in exhibiting neuroprotective and neuroproliferative activities (Nie et al., 2014). However, the neurogenic effectts of RA had not been tested in a diabetes model induced by STZ. The observed decline with T2DM in the expression of neuronal markers across all the stages of neurogenesis, i.e. proliferation (Ki67), migration(DCX) and maturation of neurons $(\mathrm{NeuN})$, indicates a defective neurogenesis process. A similar observation was reported in dietinduced insulin resistance and in a prediabetes animal model showing aberrant proliferation and neurogenesis in the CNS (Ramos-Rodriguez et al., 2014), indicative of deleterious effects by hyperglycemia. Interestingly, RA significantly enhanced the expression of the studied neuronal markers in T2DM mice, suggesting its positive influence on hippocampal neurogenesis during the disease; although the underlying mechanism(s) remains to be elucidated.

\section{CONCLUSION}

Our data provide further evidence for the cognitive deficits associated with T2DM and the importance of diabetes as a risk factor for AD. In addition, the anti- diabetic effects and the regulation of gene expression of neuronal markers by RA suggest its potential as a neuroprotective compound with promising neurogenic effects during hyperglycemia. In summary, this study proposes RA as a potential candidate to mitigate dementia by enhancing adult hippocampal neurogenesis and as a therapeutic compound for treating hyperglycemia associated with diabetes.

\section{CONFLICT OF INTEREST}

The authors declare that there are no conflicts of interest.

\section{ACKNOWLEDGEMENTS}

This research was supported through BS student's research grant by National University of Sciences and Technology (NUST), Islamabad, Pakistan. We are grateful to Dr. Antonio Currais (The Salk Institute for Biological Studies, La Jolla, San Diego, USA) for proofreading of the manuscript.

\section{REFERENCES}

Ahmed S, Mahmood Z, Zahid S. Linking Insulin with Alzheimer's Disease- Emergence as Type III Diabetes. Neurol Sci. 2015;36(10):1763-9.

Alberti KG, Zimmet PZ. Definition, diagnosis and classification of diabetes mellitus and its complications. Part 1: diagnosis and classification of diabetes mellitus. Provisional report of a WHO Consultation. Diabet Med. 1998;15(7):538-53.

Alkam T, Nitta A, Mizoguchi H, Itoh A, Nabeshima T. A natural scavenger of peroxynitrites, rosmarinic acid, protects against impairment of memory induced by A $\beta 25-35$. Behav Brain Res. 2007;180(2):139-45.

Arrieta-Cruz I, Gutiérrez-Juárez R. The role of insulin resistance and glucose metabolism dysregulation in the development of Alzheimer's disease. Rev Invest Clin. 2016;68(2):53-8.

Carvalho C, Machado N, Mota PC, Correia SC, Cardoso S, Santos RX, et al. Type 2 diabetic and Alzheimer's disease mice present similar behavioral, cognitive, and vascular anomalies. J Alzheimers Dis. 2013;35(3):623-35.

DeFronzo R. Pathogenesis of type 2 diabetes mellitus. Med Clin North Am. 2004;88(4):787-835. 
Dong J, Xu H, Wang PF, Cai GJ, Song HF, Wang CC et al. Nesfatin-1 stimulates fatty-acid oxidation by activating AMPactivated protein kinase in STZ-induced type 2 diabetic mice. PloS One. 2013;8(12):e83397.

Fonteles AA, de Souza CM, de Sousa Neves JC, Menezes AP, Santos do Carmo MR, Fernandes FD2 et. al. Rosmarinic acid prevents against memory deficits in ischemic mice. Behav Brain Res. 2015;297:91-103.

Hamaguchi T, Ono K, Murase A, Yamada M. Phenolic compounds prevent Alzheimer's pathology through different effects on the amyloid-beta aggregation pathway. Am J Pathol. 2009;175(6):2557-65.

Ito N, Yabe T, Gamo Y, Nagai T, Oikawa T, Yamada H, Hanawa T. Rosmarinic Acid from Perillae Herba Produces an Antidepressant-Like Effect in Mice through Cell Proliferation in the Hippocampus. Biol Pharm Bull. 2008;31(7):1376-80.

Khamse S, Sadr SS, Roghani M, Hasanzadeh G, Mohammadian M. Rosmarinic acid exerts a neuroprotective effect in the kainate rat model of temporal lobe epilepsy: Underlying mechanisms. Pharm Biol. 2015;53(12):1818-25.

Leger M, Quiedeville A, Bouet V, Haelewyn B, Boulouard $\mathrm{M}$, Schumann-Bard $\mathrm{P}$ et al. Object recognition test in mice. Nat Protoc. 2013;8(12):2531-37.

Li W, Zhang M, Gu J, Meng ZJ, Zhao LC, Zheng, YN et al. Hypoglycemic effect of protopanaxadiol-type ginsenosides and compound $\mathrm{K}$ on Type 2 diabetes mice induced by high-fat diet combining with streptozotocin via suppression of hepatic gluconeogenesis. Fitoterapia. 2012;83(1):192-8.

Livak KJ, Schmittgen TD. Analysis of Relative Gene Expression Data Using Real-Time Quantitative PCR and the $2_{\mathrm{T}}^{-\Delta \Delta C}$ Method. Methods. 2001;25(4):402-8.

Moroz N, Tong M, Longato L, Xu H, de la Monte SM. Limited Alzheimer-type neurodegeneration in experimental obesity and type 2 diabetes mellitus. J Alzheimer's Dis. 2008;15(1):29-44.

Nie H, Peng Z, Lao N, Wang H, Chen Y, Fang Z et al. Rosmarinic acid ameliorates PTSD-like symptoms in a rat model and promotes cell proliferation in the hippocampus. Prog Neuropsychopharmacol Biol Psychiatry. 2014;51:16-22.

Noor A, Zahid S. Alterations in adult hippocampal neurogenesis, aberrant protein s-nitrosylation, and associated spatial memory loss in streptozotocin-induced diabetes mellitus type 2 mice. Iran J Basic Med Sci. 2017;20(10): 1159-65.

Petersen M, Simmonds MS. Rosmarinic acid. Phytochemistry. 2003;62(2):121-5.

Ramos-Rodriguez JJ, Molina-Gil S, Ortiz-Barajas O, Jimenez-Palomares M, Perdomo G, Cozar-Castellano I, et al. Central proliferation and neurogenesis is impaired in type 2 diabetes and prediabetes animal models. PloS One. 2014;9(2):e89229.

Rossetti L. Glucose toxicity: the implications of hyperglycemia in the pathophysiology of diabetes mellitus. Clin Invest Med. 1995;18(4):255-60.

Sridhar GR, Lakshmi G, Nagamani G. Emerging links between type 2 diabetes and Alzheimer's disease. World J Diabetes. 2015;6(5):744-51.

Sotnikova R, Okruhlicova L, Vlkovicova J, Navarova J, Gajdacova B, Pivackova L. Rosmarinic acid administration attenuates diabetes-induced vascular dysfunction of the rat aorta. J Pharm Pharmacol. 2013;65(5):713-23.

Srinivasan K, Viswanad B, Asrat L, Kaul C, Ramarao P. Combination of high-fat diet-fed and low-dose streptozotocintreated rat: A model for type 2 diabetes and pharmacological screening. Pharmacol Res. 2005;52(4),313-20.

Tavafi M, Ahmadvand H, Tamjidipoor A, Delfan B, Khalatbari AR. Satureja khozestanica essential oil ameliorates progression of diabetic nephropathy in uninephrectomized diabetic rats. Tissue Cell. 2011;43(1):45-51.

Vagelatos NT, Eslick GD. Type 2 diabetes as a risk factor for Alzheimer's disease: the confounders, interactions, and neuropathology associated with this relationship. Epidemiol Rev. 2013;35(1):152-60.

Zhang M, Lv XY, Li J, Xu ZG, Chen L. The characterization of high-fat diet and multiple low-dose streptozotocin induced type 2 diabetes rat model. Exp Diabetes Res. 2008;2008:704045.

Received for publication on $03^{\text {rd }}$ October 2018 Accepted for publication on $11^{\text {st }}$ March 2019 